\title{
Electrostatic Analysis of Moving Conductors Using a Perturbation Finite Element Method
}

\author{
Mohamed Boutaayamou ${ }^{1}$, Ruth V. Sabariego ${ }^{1}$ and Patrick Dular ${ }^{1,2}$ \\ ${ }^{1}$ ACE, Dept. of Electrical Engineering and Computer Science, University of Liège, Belgium \\ ${ }^{2}$ Fonds de la Recherche Scientifique - FNRS, Belgium
}

This paper deals with the analysis of electrostatic problems involving moving devices by means of a perturbation finite element method. A reference problem without any moving parts is first solved and gives the source for a sequence of perturbation problems in subdomains restricted to the neighbourhood of these parts. The source accounts for all the previous calculations for preceding positions what increases the efficiency of the simulations. This proposed approach also improves the computation accuracy and decreases the complexity of the analysis of moving conductors thanks to the use of independent and adaptively refined meshes.

Index Terms_Electrostatic analysis, Finite element methods, Perturbation methods, Movement.

\section{INTRODUCTION}

$\mathbf{T}$ HE FINITE element (FE) analysis of problems involving movement often requires a completely new computation and mesh for every position of the moving part [1]. This may be computationally expensive specially when dealing with 3D models [2].

In [3], an iterative perturbation FE method (PFEM) has been used for computing electrostatic field distortions and the ensuing charges and forces appearing on moving conductive regions subjected to fixed potentials. A reference problem without moving parts is first solved and gives the source for a sequence of independent perturbation problems (one per moving part). An iterative process is then used to obtain a precise solution for relative positions when the coupling between the source and perturbing regions is significant. However, for some critical positions, many iterations may be required to ensure accuracy which increases the computational cost.

In this paper, an extension to this approach is presented. Now, the source (feeding the sequence) of perturbation problems for each position is computed considering not the reference problem at initial position (only) but exploiting instead all the previous calculations for preceding positions. The iterative process is thus accelerated and computational cost reduced. Hereafter, the considered conductors are supposed to move with small speed in the absence of any magnetic field in order to satisfy the static field assumption. The method is highlighted and validated on a test case.

\section{PFEM FOR MOVING Electrostatic CONDUCTORS}

\section{A. Canonical problem in a strong form}

An electrostatic problem $p$ is defined in a bounded domain $\Omega_{p}$ of the 2-D or 3-D Euclidean space. The subscript $p$ refers to the associated problem $p$. The domain $\Omega_{p}$ comprises fixed or moving conductive regions denoted by $\Omega_{c, p}$. The boundary of $\Omega_{p}$ is denoted by $\Gamma_{p}$ (possibly at infinity), with complementary parts $\Gamma_{e, p}$ and $\Gamma_{d, p}$, and the boundary of $\Omega_{c, p}$ by $\Gamma_{c, p}$ (Fig. 1).

The governing differential equations and constitutive law are

$$
\operatorname{curl} \boldsymbol{e}_{p}=0, \quad \operatorname{div} \boldsymbol{d}_{p}=0, \quad \boldsymbol{d}_{p}=\varepsilon_{p} \boldsymbol{e}_{p},
$$

with associated boundary conditions $(\mathrm{BCs})$

$$
\boldsymbol{n} \times\left.\boldsymbol{e}_{p}\right|_{\Gamma_{e, p}}=0,\left.\quad \boldsymbol{n} \cdot \boldsymbol{d}_{p}\right|_{\Gamma_{d, p}}=0,
$$

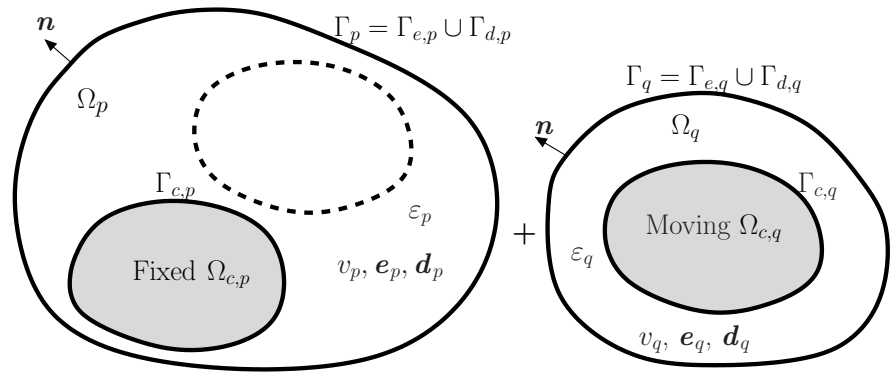

Fig. 1. Extraction of the moving conductor $\Omega_{c, q}$ from the domain $\Omega_{p}$ and the definition of a reduced subdomain $\Omega_{q}$ containing $\Omega_{c, q}$ and its surrounding.

where $\boldsymbol{e}_{p}$ is the electric field, $\boldsymbol{d}_{p}$ is the electric flux density, $\varepsilon_{p}$ is the electric permittivity and $\boldsymbol{n}$ is the unit normal exterior to $\Omega_{p}$. In (1b), we assume that there is no volume charge density in $\Omega_{p}$. From (1a), the electric field $\boldsymbol{e}_{p}$ can be derived from an electric scalar potential $v_{p}$, i.e., $\boldsymbol{e}_{p}=-\operatorname{grad} v_{p}$.

At the discrete level, independent meshes are used for all problems $p$. This allows an adapted mesh refinement of different regions and avoids any intersection conflict between the boundaries of problems $p$ defined in moving domains. The distortion of the mesh is thus avoided in case of a critical displacement or deformation of the moving regions.

\section{B. Perturbation problems}

The addition of a perturbing moving conductive region $\Omega_{c, p}$ to a reference configuration modifies the electric field distribution and leads to electric field distortions, i.e. the socalled perturbation fields.

In the FE perturbation simulation of moving conductors, $P$ successive sub-problems $p=1, \ldots, P$ are solved. The summation of their solutions gives the total solution of the complete problem as

$$
v=\sum_{p=1}^{P} v_{p}, \quad \boldsymbol{e}=\sum_{p=1}^{P} \boldsymbol{e}_{p}, \quad \boldsymbol{d}=\sum_{p=1}^{P} \boldsymbol{d}_{p} .
$$

For a significant coupling between a given sub-domain $\Omega_{p}$ and the other sub-domains, an iterative process is used to determine an accurate solution $v_{p}$ of problem $p$. In this case, $v_{p}$ is obtained as a series of corrections, i.e.

$$
v_{p}=v_{p, 1}+v_{p, 2}+v_{p, 3}+\ldots
$$

where $v_{p, i}$ is the solution of sub-problem $p, i$. The iterative 
process is repeated until convergence for a given tolerance.

As each problem $p, i$ is perturbed by all the others, its solution, i.e. correction $v_{p, i}$, must take into account the influence of all the previous corrections $v_{q, j}$ of the other sub-problems, with $q=1, \ldots, p-1 ; j=i$ and $q=p+1, \ldots, P ; j=i-1$. Further, initial solutions $v_{p, 0}$ are set to zero.

In this paper, the added perturbing moving region $\Omega_{c, p}$ is a perfect conductor. The source of each sub-problem $p, i$ is the result of the summation of all the previously calculated solutions $v_{q, j}$ and it is applied as a non-homogeneous Dirichlet $\mathrm{BC}$, i.e.

$$
\left.v_{p, i}\right|_{\Gamma_{c, p}}=-\sum_{\substack{q=1 \\ q \neq p}}^{P} v_{q, j},
$$

where $j$ is the last iteration index for which the associated solution is known.

In regions whit different $\varepsilon_{p}$, an additional source term has to be considered in (1c) [3]. Because the added region $\Omega_{c, p}$ is a perfect conductor, all the $\varepsilon_{p}$ are equal and denoted $\varepsilon$.

Fig. 2 illustrates the case of two sub-problems where subproblem $p=1$ is chosen as a reference or source problem.

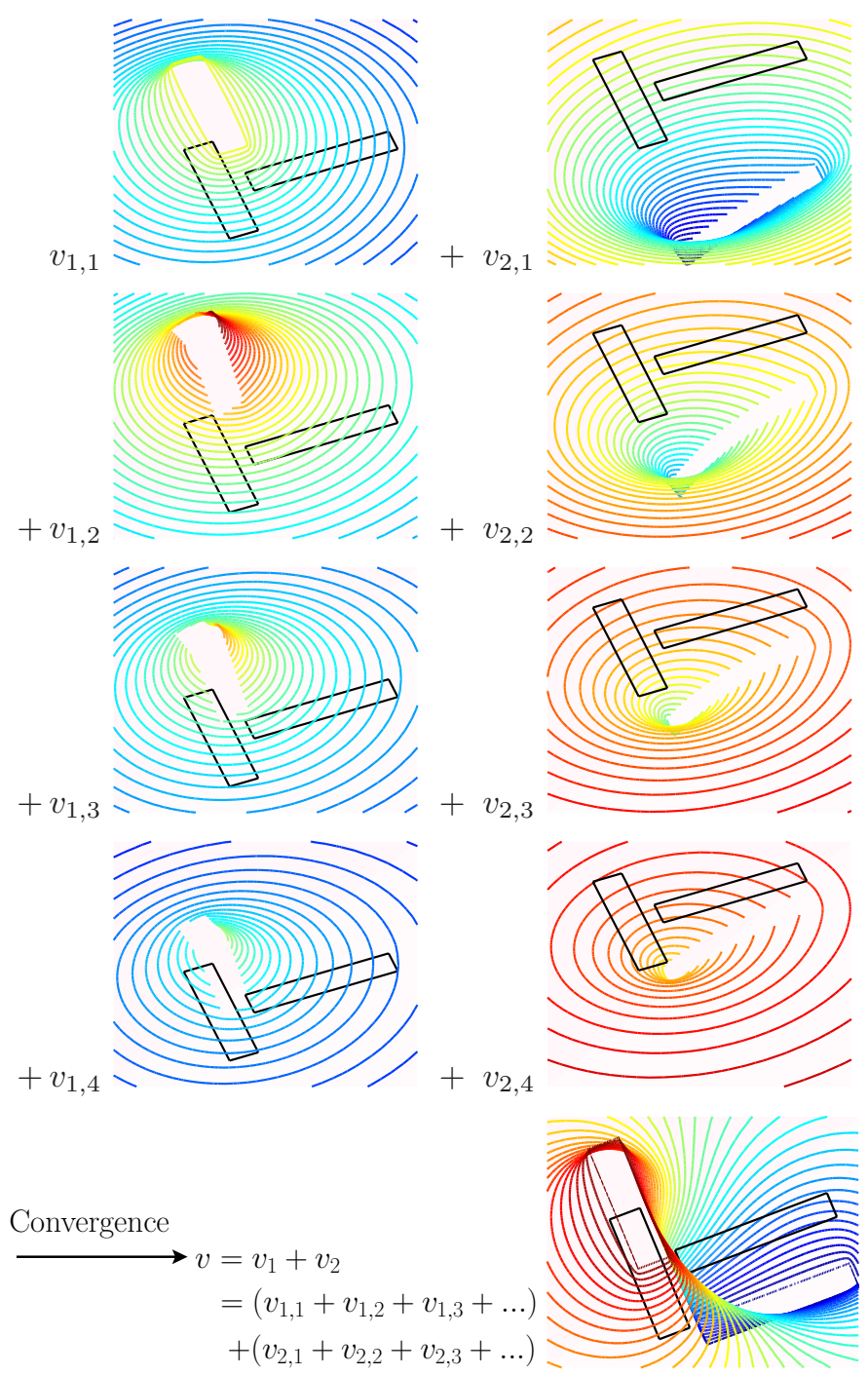

Fig. 2. The distribution of the electric potential corrections calculated in each sub-problem $\mathrm{p}=1,2$ and the total solution $v$ of the complete problem (after convergence). Sub-problem $p=1$ is chosen as the reference or source sub-problem.

\section{WEAK Finite ElEMENT Formulations}

\section{A. Canonical problem in a weak form}

The electrostatic problem $p(1 \mathrm{a}-\mathrm{c})$ can be solved as a solution of the electric scalar potential formulation obtained from the weak form of (1b), i.e., $\operatorname{div}\left(-\varepsilon \operatorname{grad} v_{p}\right)=0$, as

$$
\begin{gathered}
\left(-\varepsilon \operatorname{grad} v_{p}, \operatorname{grad} v^{\prime}\right)_{\Omega_{p}}-\left\langle\boldsymbol{n} \cdot \boldsymbol{d}_{p}, v^{\prime}\right\rangle_{\Gamma_{d, p}}=0, \\
\forall v^{\prime} \in F\left(\Omega_{p}\right),
\end{gathered}
$$

where $F\left(\Omega_{p}\right)$ is the function space defined on $\Omega_{p}$ containing the basis functions for $v_{p}$ as well as for the test function $v^{\prime}$ [4]; $(\cdot, \cdot)_{\Omega}$ and $\langle\cdot, \cdot\rangle_{\Gamma}$ denote, respectively, a volume integral in $\Omega$ and a surface integral on $\Gamma$ of products of scalar or vector fields. At the discrete level, $F\left(\Omega_{p}\right)$ is approximated with nodal FEs. The surface integral term in (6) can be associated with a global quantity or used for fixing a natural BC (usually homogeneous for a tangent electric field constraint) on a portion $\Gamma_{d, p}$ of the boundary of $\Gamma_{p}$.

Formulation (6) is valid for any correction $v_{p, i}$ in (4) involved in the iterative process. The associated BC (5) on $\Gamma_{c, p}$ has to be strongly imposed in $F\left(\Omega_{p}\right)$.

\section{B. Projection of sources}

The source of the perturbation problem $p$ is a scalar potential $v_{q}$ applied as a BC (5). Because this source is interpolated in the mesh of problem $q$, a projection method [5] is used to evaluate $v_{q}$ in the mesh of $\Omega_{p}$.

Given the perfectly conductive nature of the perturbing region $\Omega_{c, p}$, the projection $v_{q_{p r o j}}$ of $v_{q}$ can be limited to $\Gamma_{c, p}$. It reads

$$
\begin{gathered}
\left\langle\operatorname{grad} v_{q_{p r o j}}, \operatorname{grad} v^{\prime}\right\rangle_{\Gamma_{c, p}}-\left\langle\operatorname{grad} v_{q}, \operatorname{grad} v^{\prime}\right\rangle_{\Gamma_{c, p}}=0, \\
\forall v^{\prime} \in F\left(\Gamma_{c, p}\right),
\end{gathered}
$$

where the function space $F\left(\Gamma_{c, p}\right)$ contains $v_{q_{p r o j}}$ and its associated test function $v^{\prime}$. At the discrete level, $v_{q_{p r o j}}$ is discretized with nodal FEs and is associated to a gauge condition fixing a nodal value in $\Gamma_{c, p}$. For sake of simplicity, $v_{q_{\text {proj }}}$ will be referred to as $v_{q}$.

Further, the projection is to be extended to the whole domain $\Omega_{c, p}$ in case of a dielectric perturbing region. We choose to directly project $\operatorname{grad} v_{q}$ in order to assure a better numerical behaviour in the ensuing equations where the involved quantities are also gradients.

When all components of previously calculated electric field sources $\boldsymbol{e}_{q}=-\operatorname{grad} v_{q}$ are needed (to give access also to normal gradients) in the layer of FEs touching $\Gamma_{c, p}$ in $\Omega_{p} \backslash \Omega_{c, p}$, denoted $\Omega_{l, p}$, the projection (7) of $v_{q}$ has to be extended only to this transition layer. This way, the computational effort of the projection is also reduced. Having access to $\boldsymbol{e}_{p}$ and $\boldsymbol{e}_{q}$ in this layer allows to compute there the perturbed electric field $\boldsymbol{e}$. Both charges and electric forces can thus be calculated on $\Gamma_{c, p}$.

Formulation (6) has to account for non-homogeneous Neumann $\mathrm{BC}$ as well, i.e.

$$
\left.\boldsymbol{n} \cdot \boldsymbol{d}_{p, i}\right|_{\Gamma_{d, p}}=-\sum_{\substack{q=1 \\ q \neq p}} \boldsymbol{n} \cdot \boldsymbol{d}_{q, j},
$$

The latter is not known in a strong sense. The associated 
surface integral term in (6) can be evaluated via the weak formulation of problems $q, j$ now applied to $\Omega_{l, p}$, as

$$
\begin{aligned}
\left\langle\boldsymbol{n} \cdot \boldsymbol{d}_{p, i}, v^{\prime}\right\rangle_{\Gamma_{d, p}} & =-\left\langle\sum_{\substack{q=1 \\
q \neq p}} \boldsymbol{n} \cdot \boldsymbol{d}_{q, j}, v^{\prime}\right\rangle_{\Gamma_{d, p}} \\
& =-\left(-\varepsilon \operatorname{grad} \sum_{\substack{q=1 \\
q \neq p}} v_{q, j}, \operatorname{grad} v^{\prime}\right)_{\Omega_{l, p}}, \\
& \forall v^{\prime} \in F\left(\Omega_{l, p}\right),
\end{aligned}
$$

benefiting from the already known projection of $v_{q, j}(7)$.

\section{Electrostatic charges}

A suitable treatment of the surface integral term in (6) consists in naturally defining a global electric charge in the weak sense [4]. A test function $v_{c, p}$ is chosen equal to one on $\Gamma_{c, p}$ and continuously varying towards 0 in the layer $\Omega_{l, p}$ of FEs touching $\Gamma_{c, p}$ in $\Omega_{p} \backslash \Omega_{c, p}$.

Each solution $v_{p, i}$ calculated in (6) and the projected $v_{q, j}$ (7) lead to an electric charge correction $Q_{p, i}$ appearing on $\Gamma_{c, p}$ in iteration $i$. This charge can be naturally obtained at the post-processing stage through the volume integral in (6) limited to $\Omega_{l, p}$, i.e.,

$$
\begin{aligned}
Q_{p, i}= & -\left(-\varepsilon \operatorname{grad} v_{p, i}, \operatorname{grad} v_{c, p}\right)_{\Omega_{l, p}} \\
& -\left(-\varepsilon \operatorname{grad} \sum_{\substack{q=1 \\
q \neq p}}^{P} v_{q, j}, \operatorname{grad} v_{c, p}\right)_{\Omega_{l, p}}
\end{aligned}
$$

Further, the total electric charge appearing on $\Gamma_{c, p}$ is given by

$$
Q_{p}=\sum_{i \geq 1} Q_{p, i}
$$

\section{Electrostatic forces}

As previously mentioned, the perturbed electric field $\boldsymbol{e}$ can be computed in the transition layer $\Omega_{l, p}$ of FEs touching $\Gamma_{c, p}$ in $\Omega_{p} \backslash \Omega_{c, p}$. The electric force distribution is calculated thus by locally applying the virtual work principle [6] in $\Omega_{l, p}$. At the discrete level, the force at each node of $\Gamma_{c, p}$ is obtained by deriving the electric energy in the considered layer of FEs with respect to a virtual displacement. The contribution of a reference element $\Delta$ to the force in a given direction is

$$
F_{r}=\int_{\Delta}\left(-\varepsilon \boldsymbol{e} J^{-1} \frac{\partial J}{\partial u} \boldsymbol{e}+\boldsymbol{e} \boldsymbol{e} \frac{\partial|J|}{\partial u}\right) d \Delta,
$$

for a virtual displacement $r$ in this direction. $J$ is the geometrical Jacobian matrix with determinant $|J|$.

Given the non-linearity of the force, a direct summation of the forces at each iteration is not possible. The total electric field $\boldsymbol{e}$ has to be updated at each iteration before computing the total force (12).

\section{E. Taking movement into account}

Up to now, the first position of a moving perturbing conductor $\Omega_{c, p}$ has been considered. Now, the electrostatic analysis will be carried out for its next positions.

In the PFEM simulation, the position of the moving conductive region $\Omega_{c, p}$ is either a given function of time or obtained from solving an equation of motion that determines the displacements of $\Omega_{c, p}$. In the latter case, a weak electromechanical coupling is considered where the electric forces are the input forces for the mechanical problem. Hereafter, the electrical problem is solved step-by-step using the implicit Euler method, which is unconditionally stable.

At the initial time $t_{0}$, i.e. the first position, a problem $k$ of the form $(1 \mathrm{a}-\mathrm{c})$ is considered and called the reference or source problem. Its solution $v_{k}$ given by (4) will be exploited hereafter.

At $t_{1}=t_{0}+\Delta t$, the conductor $\Omega_{c, p}$ moves and the total solution $v_{k}$ previously calculated (at $t_{0}$ ) improves the source of problem $p$ at iteration 1 as

$$
\left.v_{p, 1}\right|_{\Gamma_{c, p}}=-v_{k}-\sum_{\substack{q=1 \\ q \neq p, k}}^{P} v_{q, j} .
$$

The improvement of this source is obvious since $v_{k}$ accounts for all the previous calculations done in the first position. The solution of perturbation problem $p, 1$ is now closer to the suitable correction in $\Omega_{p}$ than that calculated in $t_{0}$. The number of iterations to achieve the convergence of $v_{p}$ is thus reduced.

Next, we consider the reference problem $k$ at iteration $i=2$. The source $v_{k}$ that fed problem $p$, has to be counterbalanced at this iteration. That is because the non-homogeneous Dirichlet BC of problem $k$ has to be corrected. This is done by subtracting the quantity $v_{k}-v_{k, 1}$ from the other projected solutions $v_{q, j}$, i.e.

$$
\left.v_{k, 2}\right|_{\Gamma_{c, p}}=-\left(\sum_{\substack{q=1 \\ q \neq k}}^{P} v_{q, j}-\left(v_{k}-v_{k, 1}\right)\right) .
$$

For next iterations $i \geq 2$, the perturbation problems $p$ are solved analogously. The iterative process is repeated until convergence for a given tolerance.

The same reasoning of the resolutions holds for the next time step.

\section{ApPliCATION}

As example, an electrostatic actuated combdrive is considered. The geometry of its unit cell is shown in Fig. 3 ( $L=10 \mu \mathrm{m}, b=2 \mu \mathrm{m}$ and $g=2 \mu \mathrm{m}$ ). Applying a voltage difference between the comb structures will result in the displacement of its movable part by electrostatic forces. This movement is simulated here by varying the finger engagement denoted as $x$.

The reference problem is defined in the domain $\Omega_{1}$ surrounding the fixed comb (i.e., $\Omega_{c, 1}$ ). The latter is coarsely meshed (Fig. 4 (right)). The domain $\Omega_{2}$ containing the moving finger (i.e., $\Omega_{c, 2}$ ) has an adapted mesh especially fine in the vicinity of the corners (Fig. 4 (left)). The fringing field effects are thus precisely taken into account and the electric forces are accurately calculated. The two meshes are independent what allows for any intersection of the perturbation problem boundaries with the reference problem region materials. The distortion of the mesh is also avoided in case of a critical displacement of the moving finger.

The electric charges appearing on the surface of the comb structures and the outer surface of $\Omega_{1}$ are calculated by both 


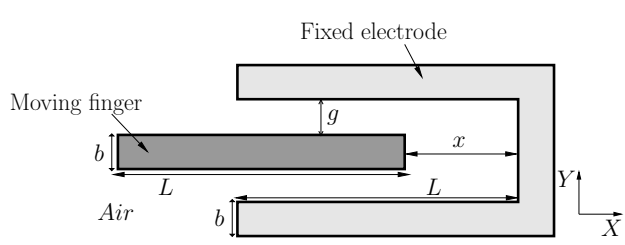

Fig. 3. Geometry of a unit cell of a combdrive with a moving finger and fixed electrode.
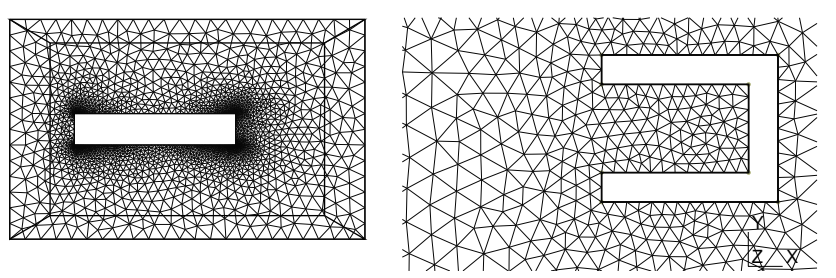

Fig. 4. Mesh of $\Omega_{1}$ (right) and adapted mesh of $\Omega_{2}$ with infinite boundaries around the moving finger (left).

the FE perturbation technique and the conventional FEM. The numerical results are compared in Fig. 5. A good agreement is observed. In addition, the charge conservation of the whole system is shown.

The electric forces on the outer surface of the moving finger are also computed. In order to compare with the FE solution, we consider the summation of the values of the $x$-component of the electric forces at each node of both left and right side of the mover (Fig. 6). The numerical results agree well (the relative error with respect to the FEM results reaches $3 \%$ with relative accuracy $1 \%$ ).

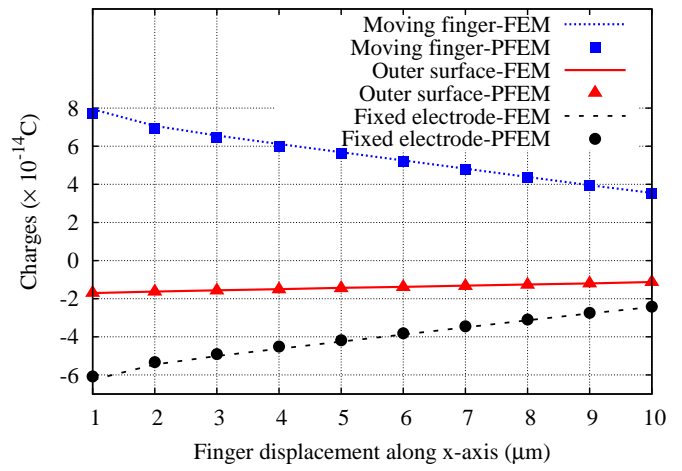

Fig. 5. Electric charges versus finger displacement.

In Fig. 7 the number of iterations to achieve convergence (with the relative error of the electric potential below 1\%) using the proposed approach is divided by a factor 2 in comparison with what was presented in [3] where previous calculations for preceding positions are not exploited. Less iterations are then required in case of critical positions where the moving finger is close to the anchored electrode.

Furthermore, it has been verified that the PFEM solves this 2-D problem with a speed-up factor of 3 in comparison to the classical FEM.

\section{CONCLUSION}

The perturbation finite element method proposed is suitable for modeling electrostatic conductors involving movement. The mover is discretized in a reduced sub-domain with a mesh that keeps its initial quality throughout the whole movement even for a critical displacement. The complexity of the

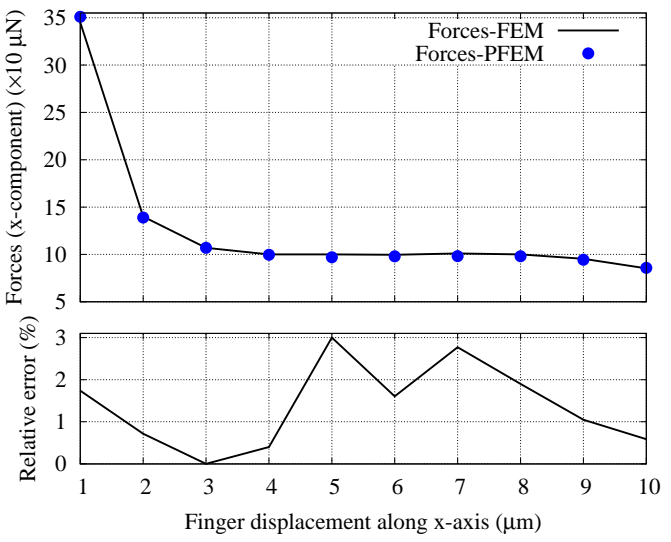

Fig. 6. Electric forces versus finger displacement (up). Relative error with regard to the conventional FEM (down).

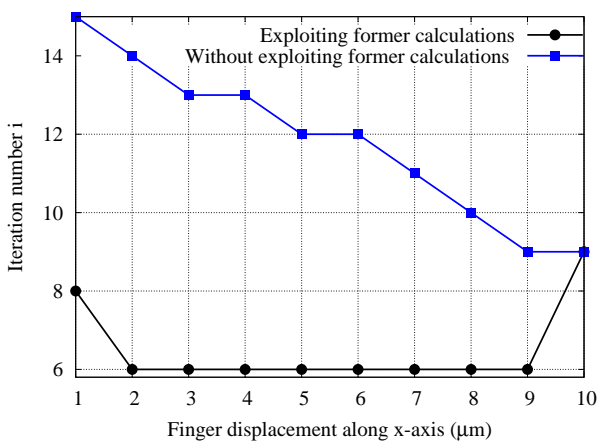

Fig. 7. Number of iterations to achieve convergence.

problem is also decreased by considering independent and adaptively refined meshes for each subproblem.

An electrostatic analysis of a combdrive involving rigid movement has been carried out. However, the proposed method applies equally to the deformation of an elastic body. The example shows that a significant speed-up is achieved and the accuracy is improved in comparison with the classical FEM calculations.

\section{ACKNOWLEDGMENT}

This work is supported by the Belgian French Community (ARC 03/08-298) and the Belgian Science Policy (IAP P6/21).

\section{REFERENCES}

[1] R. Perrin-Bit and J. L. Coulomb, "A three dimensional finite element mesh connection for problems involving movement", IEEE Trans. on Magn., vol. 31, no. 2, pp. 1920-1923, May 1995.

[2] H. C. Lai, D. Rodger, and P. C. Coles, "A 3-D Overlapping FiniteElement Scheme for Modeling Movement", IEEE Trans. on Magn., vol. 40, no. 2, pp. 533-536, Mar. 2004.

[3] M. Boutaayamou, R. V. Sabariego, and P. Dular, "An Iterative Finite Element Perturbation Method for Computing Electrostatic Field Distortions", IEEE Trans. on Magn., 2008, in press.

[4] P. Dular, W. Legros, A. Nicolet, "Coupling of local and global quantities in various finite element formulations and its application to electrostatics, magnetostatics and magnetodynamics", IEEE Trans. Magn., vol. 34, no. 5 , pp. 3078-3081, 1998.

[5] C. Geuzaine, B. Meys, F. Henrotte, P. Dular, W. Legros, "A Galerkin projection method for mixed finite elements", IEEE Trans. Magn., vol. 35, no. 3, pp. 1438-1441, 1999.

[6] J. L. Coulomb and G. Meunier, "Finite element implementation of virtual work principle for magnetic or electric force and torque computation", IEEE Trans. Magn., vol. 20, no. 5 , pp. 1894-1896, 1984.

Manuscript received May 10, 2008. Corresponding author: Mohamed Boutaayamou (e-mail: mboutaayamou@ulg.ac.be). 